\title{
Neural Activity in the Human Brain Relating to Uncertainty and Arousal during Anticipation
}

\author{
Hugo D. Critchley, ${ }^{\star} † \S$ Christopher J. Mathias, \\ and Raymond J. Dolan* \\ *Wellcome Department of Cognitive Neurology \\ 12 Queen Square \\ Institute of Neurology \\ University College London \\ London WC1N 3BG \\ United Kingdom \\ †Autonomic Unit \\ National Hospital for Neurology and Neurosurgery \\ London WC1N 3BG \\ United Kingdom \\ ¥Neurovascular Medicine Unit \\ St. Mary's Hospital \\ Imperial College School of Medicine \\ London W2 1NY \\ United Kingdom
}

\section{Summary}

We used functional magnetic resonance neuroimaging to measure brain activity during delay between reward-related decisions and their outcomes, and the modulation of this delay activity by uncertainty and arousal. Feedback, indicating financial gain or loss, was given following a fixed delay. Anticipatory arousal was indexed by galvanic skin conductance. Delayperiod activity was associated with bilateral activation in orbital and medial prefrontal, temporal, and right parietal cortices. During delay, activity in anterior cingulate and orbitofrontal cortices was modulated by outcome uncertainty, whereas anterior cingulate, dorsolateral prefrontal, and parietal cortices activity was modulated by degree of anticipatory arousal. A distinct region of anterior cingulate was commonly activated by both uncertainty and arousal. Our findings highlight distinct contributions of cognitive uncertainty and autonomic arousal to anticipatory neural activity in prefrontal cortex.

\section{Introduction}

Adaptive behavior requires an ability to make advantageous decisions by predicting the likelihood of future success based upon previous experience. Lesions to such brain regions as ventral and medial prefrontal cortex as well as anterior medial temporal lobes can result in disturbed social and emotional behavior, which is associated with abnormalities in strategic decisionmaking (Eslinger and Damasio, 1985; Damasio et al., 1990; Shallice and Burgess 1991; Bechara et al., 1997; Adolphs et al., 1998). Characteristically, individuals with ventromedial/orbitofrontal lesions fail to adapt their responses to new reinforcement contingencies and show behavioral perseveration (Rolls et al., 1994). When given

§ To whom correspondence should be addressed (e-mail: h.critchley@ fil.ion.ucl.ac.uk). the choice between high-risk decisions potentially offering immediate rewards and low-risk decisions resulting in long-term gain but little short-term reward, subjects with ventromedial prefrontal lesions select the high-risk strategy (Bechara et al., 1996). In contrast, intact subjects adopt the more advantageous low-risk strategy with learning (Bechara et al., 1997, 1999). With learning, normal subjects develop anticipatory sympathetic arousal before selecting high-risk options; this anticipatory arousal is absent in patients with ventromedial prefrontal damage, who are indifferent to risk despite normal autonomic responses to such "physical" stimuli as loud noises (Bechara et al., 1996; Tranel, 2000). These patients have reduced arousal responses (indexed by galvanic skin conductance responses [GSR]) to other "psychological" stimuli (e.g., affective pictures) (Tranel and Damasio, 1994; Bechara et al., 1999; Tranel, 2000).

The observation of a conjunction between absent anticipatory arousal and maladaptive behavioral strategies has led to a hypothesis that cerebral representation of anticipatory arousal biases behavior and guides strategic decision-making (Damasio et al., 1991; Damasio, 1994; Bechara et al., 1996). This behavioral bias may be dissociated from explicit, conscious knowledge about risk. Healthy subjects show a preference for low-risk decisions and show increased amplitude of anticipatory skin-conductance responses to high-risk options before developing explicit awareness of the most advantageous behavioral strategy (Bechara et al., 1997). Moreover, patients with ventromedial prefrontal cortical lesions may demonstrate explicit awareness of the risks associated with particular decisions but are unable to modify their behavior accordingly (Saver and Damasio, 1991; Bechara et al., 1997). Thus, anticipatory arousal is an index of implicit risk-related learning that may directly influence behavior.

We used functional magnetic resonance (fMRI) to measure brain activity in healthy subjects performing a two-choice decision-making task, during which subjects responded to repeated trials of visually presented playing cards (Figure 1) by indexing whether they predicted the next card would be higher or lower. A delay period followed this decision and was followed by presentation of a feedback card that indicated whether the preceding decision had been correct (with monetary gain) or incorrect (monetary loss). Note that in this paradigm, each decision varied with respect to its associated degree of outcome uncertainty. Thus, if the face value of the cue card was 1 , subjects could predict with certainty that the feedback card would be higher. Similarly, if the face value of the cue card was 10 , the subject could predict with certainty that the ensuing feedback card would be lower. By contrast, high uncertainty was associated with presentation of cue card face values of 5 and 6, where predictions of the feedback card being (respectively) higher or lower were associated with a $44 \%$ likelihood of being wrong and a subsequent financial loss (Table 1). Using this paradigm, we were able to measure brain activity that incorporated anticipation of outcome (a component of activity during delay epoch 


\section{Task design}

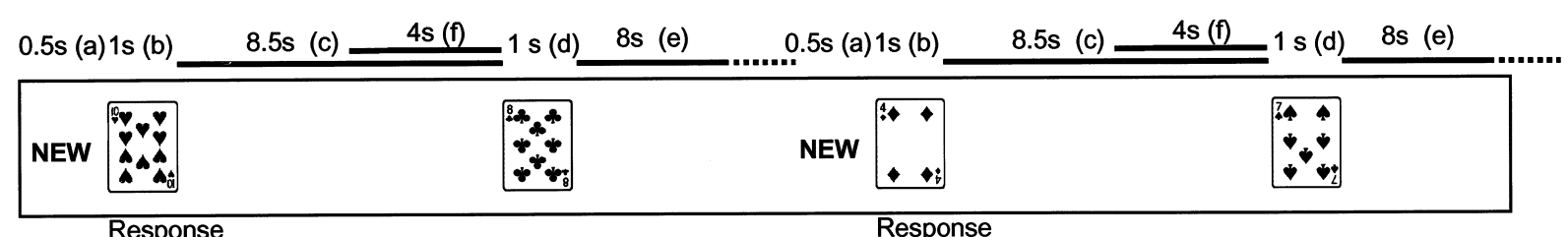

\section{Trial A: 0\% risk}

Figure 1. Experimental Task

Schematic shows two trials of the experimental task. On repeated trials, stimuli were visually presented to subjects as follows: (a) a cue for the trial (presentation of word NEW); (b) presentation of a cue card, to which the subject responded with a two-choice button press as to whether the next card would be higher or lower; (c) an $8.5 \mathrm{~s}$ anticipatory delay period before feedback; (d) feedback card indicating to subjects if their decision was right or wrong, associated with financial gain or loss; (e) intertrial interval. The data analyses explored brain activity during the delay period, and its modulation by uncertainty and arousal. Uncertainty (risk) of the decision was a function of the face value of the cue card (Table 1). Arousal was determined from mean (normalized) skin conductance over the $4 \mathrm{~s}$ period (f) prior to feedback. Thus Trial $A$ is associated with no risk as face value of the feedback card must be lower than a 10 . Trial $B$ is associated with $33 \%$ chance of the feedback card being lower than a 4.

that also included nonspecific effects such as attention) and, more critically, the modulation of anticipatory activity by uncertainty and autonomic arousal (GSR).

\section{Results}

\section{Task Performance}

Subjects, debriefed after scanning, reported that they aimed to maximize monetary gain by achieving maximum correct responses, and quickly deduced that the face values of the cue cards reflected the chance of winning or losing money. Thus, few responses were made to the less likely of the two response selections of the cue card. Consistent with this probabilistic pattern of decision making, subjects made on average 23.6 incorrect decisions over the 100 trials (optimal performance would predict errors in 20 of 100 trials).

Main Effects of Delay Period (Anticipatory Epoch) The $8.5 \mathrm{~s}$ delay period between cue card decision and presentation of feedback was associated with widespread bilateral activity in ventral and medial prefrontal

\begin{tabular}{|c|c|c|c|}
\hline $\begin{array}{l}\text { Face Value of } \\
\text { Cue Card }\end{array}$ & $\begin{array}{l}\text { Probability of } \\
\text { Next Card } \\
\text { Being Higher }\end{array}$ & $\begin{array}{l}\text { Probability of } \\
\text { Next Card } \\
\text { Being Lower }\end{array}$ & $\begin{array}{l}\text { Risk Value of } \\
\text { Cue Card }\end{array}$ \\
\hline 1 & 1 & 0 & 0 \\
\hline 2 & 0.89 & 0.11 & 11 \\
\hline 3 & 0.78 & 0.22 & 22 \\
\hline 4 & 0.67 & 0.33 & 33 \\
\hline 5 & 0.56 & 0.44 & 44 \\
\hline 6 & 0.44 & 0.56 & 44 \\
\hline 7 & 0.33 & 0.67 & 33 \\
\hline 8 & 0.22 & 0.78 & 22 \\
\hline 9 & 0.11 & 0.89 & 11 \\
\hline 10 & 0 & 1 & 0 \\
\hline
\end{tabular}

cortex, lateral and anterior temporal lobe, and right dorsolateral prefrontal cortex and inferior parietal lobe. The most significant effect was seen bilaterally in inferior prefrontal regions, including orbitofrontal cortex, extending posteriorly and superiorly into anterior insula, and inferior frontal gyrus (peaks of inferior/orbital prefrontal activity: right side, coordinates, 42, 34, -14, Z score $=7.7, p<0.05$, corrected; left side, coordinates $-54,20,10, Z$ score $=7.28, p<0.05$, corrected) (Table 2; Figure 2). Note that this delay period activity reflects, in addition to reward anticipation, a number of cognitive processes that include contributions from working memory for the cue card and response, vigilance for and anticipation of the ensuing feedback card, cumulative assessment of task progress, and non-specific eye movements. The questions of interest in this study, however, pertained to the modulation of delay period activity by uncertainty and arousal.

\section{Modulation of Delay Period Activity by Uncertainty (Risk)} To determine the influence of uncertainty, we tested for a parametric modulation of activity during the delay epoch by the degree of uncertainty associated with the preceding decision in response to the cue card. No regions were found to reach significance $(p<0.05)$ when corrected for whole brain. Lesion data, however, suggest that medial prefrontal and orbitofrontal cortices are involved in processing risk-related information during anticipation, which is necessary for guidance of future behaviors (e.g., Eslinger and Damasio, 1985; Bechara et al., 1996). Furthermore, previous functional imaging studies have implicated lateral orbitofrontal cortex in generic risk-taking processes and anterior cingulate cortex in processing relative risk in reward-related decisions (Rogers et al., 1999; Elliott et al., 2000b). We therefore used small volume corrections for these regions 


\begin{tabular}{|c|c|c|c|}
\hline Brain Region & Coordinates & Cluster Size & Z Score \\
\hline \multicolumn{4}{|c|}{ Main Effect of Delay Period ${ }^{a}$} \\
\hline \multicolumn{4}{|c|}{ Orbitofrontal cortex/anterior insula } \\
\hline Right & $42,34,-14$ & 225 & 7.70 \\
\hline \multicolumn{4}{|c|}{ Orbitofrontal cortex } \\
\hline Right & $-48,40,-12$ & 321 & 7.47 \\
\hline \multicolumn{4}{|c|}{ Inferior frontal gyrus/anterior insula } \\
\hline Left & $-54,20,10$ & 56 & 6.88 \\
\hline \multicolumn{4}{|c|}{ Middle temporal gyrus } \\
\hline Right & $56,-28,-12$ & 58 & 6.86 \\
\hline \multicolumn{4}{|l|}{ Temporal pole } \\
\hline Left & $-36,8,-34$ & 65 & 6.42 \\
\hline \multicolumn{4}{|c|}{ Inferior temporal gyrus } \\
\hline Right & $-46,-2,-32$ & 179 & 6.28 \\
\hline \multicolumn{4}{|l|}{ Temporal pole } \\
\hline Right & $38,10,-32$ & 43 & 6.12 \\
\hline \multicolumn{4}{|c|}{ Dorsolateral prefrontal cortex } \\
\hline Right & $32,10,50$ & 22 & 6.06 \\
\hline \multicolumn{4}{|c|}{ Inferior parietal lobule } \\
\hline Right & $48,-40,46$ & 32 & 5.89 \\
\hline \multicolumn{4}{|c|}{ Inferior temporal gyrus } \\
\hline Right & $46,-2,-26$ & 23 & 5.87 \\
\hline \multicolumn{4}{|c|}{ Inferior temporal gyrus } \\
\hline Right & $54,-16,-26$ & 48 & 5.85 \\
\hline \multicolumn{4}{|c|}{ Medial prefrontal cortex } \\
\hline Left & $-4,40,30$ & 78 & 5.83 \\
\hline \multicolumn{4}{|c|}{ Medial prefrontal cortex } \\
\hline Right & $12,24,48$ & 10 & 5.31 \\
\hline \multicolumn{4}{|c|}{ Modulation of Delay Period Activity by Risk } \\
\hline \multicolumn{4}{|c|}{ Anterior cingulate ${ }^{b}$} \\
\hline Right & $8,22,28$ & 59 & 4.44 \\
\hline Left & $-6,28,20$ & 25 & 3.90 \\
\hline \multicolumn{4}{|c|}{ Orbitofrontal cortex ${ }^{b}$} \\
\hline Right & $30,24,-20$ & 48 & 3.80 \\
\hline Left & $-32,14,-4$ & 10 & 3.62 \\
\hline \multicolumn{4}{|c|}{ Modulation of Delay Period Activity by Arousal, Independent of Risk ${ }^{c}$} \\
\hline \multicolumn{4}{|c|}{ Dorsolateral prefrontal cortex } \\
\hline Right & $30,42,26$ & 119 & 6.27 \\
\hline \multicolumn{4}{|c|}{ Anterior cingulate } \\
\hline Left & $4,32,24$ & 29 & 6.23 \\
\hline \multicolumn{4}{|c|}{ Modulation of Delay Period Activity by Both Arousal and Risk ${ }^{d}$} \\
\hline \multicolumn{4}{|c|}{ Anterior cingulate } \\
\hline Right & $8,28,24$ & 16 & 6.05 \\
\hline Left & $-6,28,20$ & 12 & 5.71 \\
\hline
\end{tabular}

(Worsley et al., 1996), constraining our analyses to a priori regions $(10 \mathrm{~mm}$ spheres centered on homologous areas of orbitofrontal cortex, coordinates $33,15,-12$, and $-33,15,-15$ [Elliott et al., 2000b]; $16 \mathrm{~mm}$ sphere centered on anterior cingulate coordinates $-8,28,20$ [Rogers et al., 1999]). Using this approach, delay period activity reaching corrected significance was observed to increase as a function of risk in bilateral anterior cingulate and lateral orbitofrontal cortices $(p<0.05$, corrected) (Figure 3; Table 2).

Modulation of Delay Period Activity by Arousal We next examined regional activity during the delay epoch for a parametric modulation as a function of the degree of arousal, indexed by mean GSR in the last 4 $s$ of the delay epoch. This accommodated a delay between central neural activity and peripheral GSR response, and provided consistency with other studies of anticipatory arousal (Bechara et al., 1996, 1997). In this analysis, activity in right dorsolateral prefrontal cortex and right anterior cingulate increased significantly as a function of increasing anticipatory GSR ( $p<0.05$ corrected) (Figure 4; Table 2).

\section{Modulation of Delay Period Activity by Both Uncertainty and Arousal}

To determine regions that were conjointly activated in each subject by uncertainty and arousal in the period 

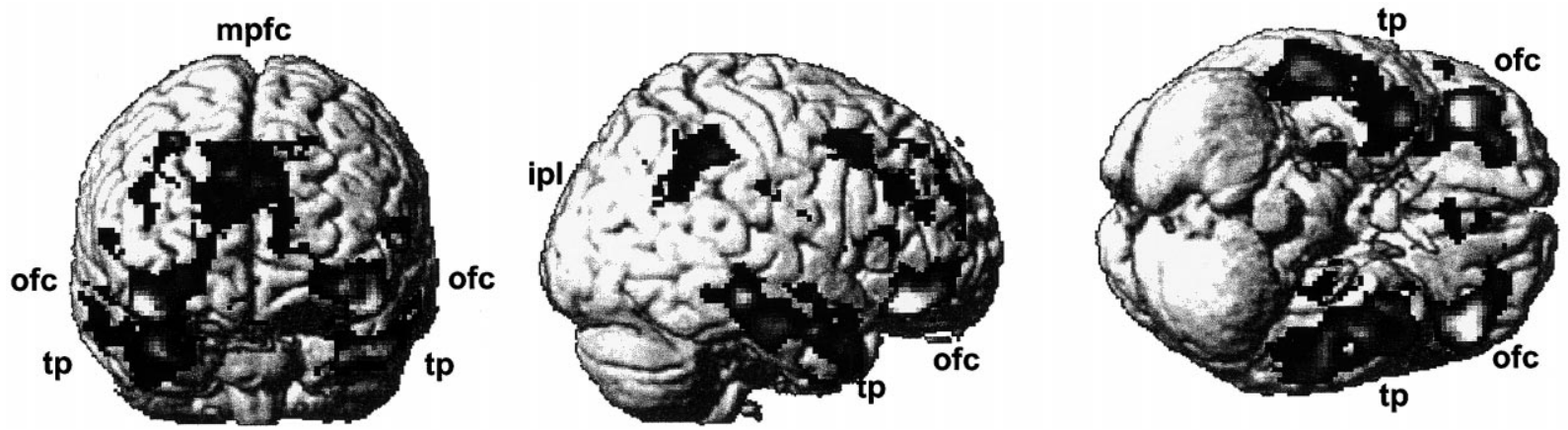

Figure 2. Activity Relating to Main Effect of Delay

Activity during the delay period before feedback may reflect a number of specific and nonspecific processes including attention, anticipation of reward-related feedback, working memory, and timing. Conjunction analysis between subjects was used to determine where significant activity ( $p<0.001$, uncorrected for illustration purposes) was expressed in all subjects. Data is plotted on a rendered template brain. Areas highlighted are bilateral orbitofrontal cortex (ofc), temporal pole (tp), medial prefrontal cortex (mpfc), and right parietal lobe (ipl).

prior to feedback, we used a conjunction analysis (see Experimental Procedures). In all subjects, bilateral regions of the anterior cingulate showed common areas of increased activity, associated with both risk and arousal ( $p<0.05$, corrected) (Figure 5; Table 2).

\section{Brain Regions Mediating Interactions between} Uncertainty and Arousal

In a final analysis, we tested for regional activity reflecting interactions between uncertainty and arousal to identify brain regions where activity was modulated by both risk and arousal in combination but not separately. No brain regions met corrected significance during the anticipatory epoch for these interactions.

\section{Discussion}

This study addressed the functional neuroanatomy of outcome anticipation by examining brain activity before outcome feedback in a reward-related decision task. a) Anterior cingulate cortex

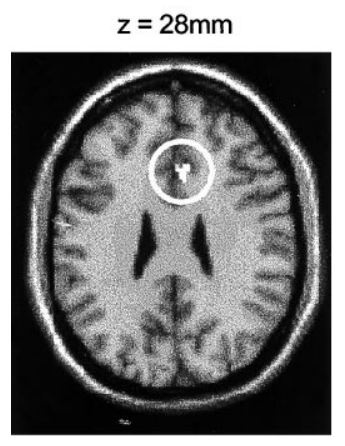

Figure 3. Modulation of Anticipatory Delay Period Activity by Risk

The figure illustrates (a) anterior cingulate cortex and (b) right anterior insula where activity during delay was modulated by decision uncertainty. Conjunction analysis across subjects was used to highlight activations common to all subjects. Spatial locations of group activity $(p<0.001$, uncorrected for illustration) are displayed on horizontal sections of a normalized template structural scan. Vertical distance from AC-PC line is given as $Z$ coordinates above the sections. Adjacent to each section is a plot of how activity in this region was modulated by the degree of uncertainty. The adjusted response of the maximally activated voxel was extracted from time-series data. The mean response for the hrf-convolved boxcar representing delay period activity is plotted against the degree of uncertainty of outcome, a function of the face value of the cue card (see Table 1). Different symbols denote responses in the different subjects.

\section{b) Orbitofrontal cortex/anterior insula}

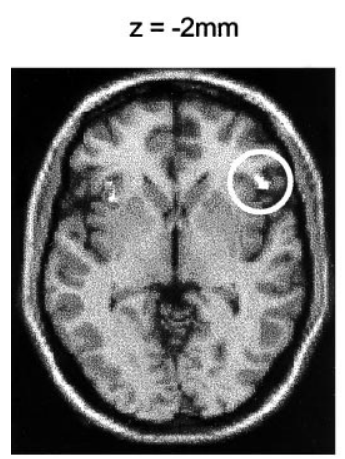

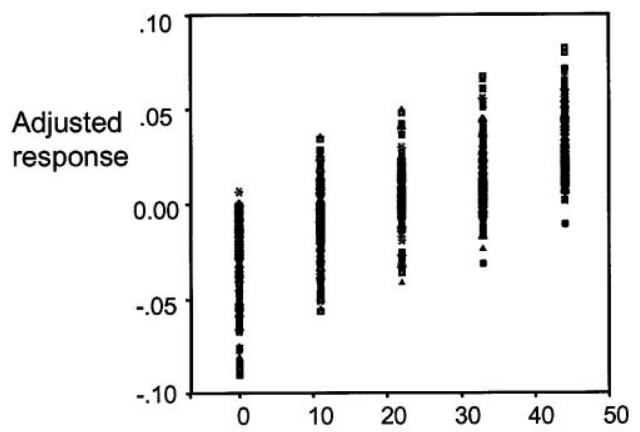

Degree of uncertainty 
a) Right dorsolateral prefrontal cortex activity positively covarying with anticipatory GSR
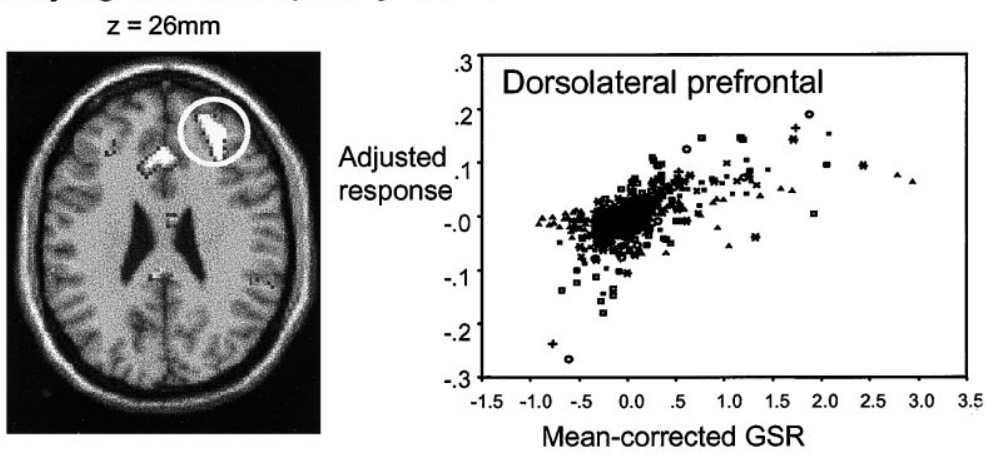

b) Right anterior cingulate activity positively co-varying with anticipatory GSR
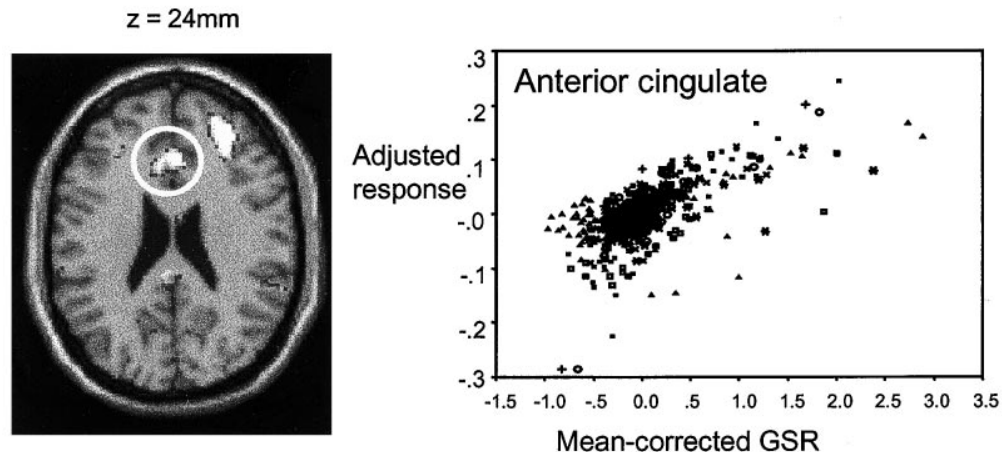

Figure 4. Modulation of Delay Period Activity by Anticipatory Autonomic Arousal

Highlighted regions reflect loci where activity in all subjects was modulated by autonomic arousal, indexed by SSR in the $4 \mathrm{~s}$ before feedback. The figure illustrates (a) right dorsolateral prefrontal and (b) anterior cingulate activity on horizontal sections of a normalized template scan, at $\mathrm{p}<0.001$ (uncorrected). Vertical distance from AC-PC line is given as $z$ coordinates above the horizontal sections. Adjacent to each section is plotted the adjusted responses of the maximally activated voxel for each region, related to the measure of anticipatory arousal (i.e., the average [mean-corrected] SSR), in the $4 \mathrm{~s}$ period prior to presentation of the feedback card. Different symbols denote responses in the different subjects.
The emphasis of the study was modulation of anticipatory delay period neural activity by outcome uncertainty and GSR. Modulation of delay period activity by cognitive uncertainty (or risk) was associated with increased activity in bilateral regions of anterior cingulate and lateral orbitofrontal cortex. Modulation of delay period activity by anticipatory autonomic arousal, indexed by GSR, was associated with enhanced activity in right dorsolateral prefrontal and anterior cingulate cortices. A discrete subregion of anterior cingulate showed effects common to degree of risk and degree of arousal, supporting a view that anterior cingulate function reflects integration of cognitive states with adaptive changes in bodily states mediated by the autonomic nervous system (Critchley et al., 2000b).

Galvanic skin conductance response can be used to index representations of outcome uncertainty and implicit learning of reward contingencies (Damasio et al., 1991; Bechara et al., 1997, 1999). Healthy individuals show increases in GSR prior to making risky decisions and adapt their risk-taking behavior with experience, while patients with orbitofrontal lesions do not show these anticipatory increases in GSR (Bechara et al., 1996, 1999; Tranel, 2000). Patients with orbitofrontal lesions also show insensitivity to punishment, and maintain disadvantageous decision-making strategies where high-risk options offering the possibility of greater shortterm reward are preferred to low-risk options with greater long-term advantage (Damasio et al., 1990, 1991;
Damasio, 1994; Bechara et al., 1996, 1997, 1999). The association between anticipatory autonomic arousal and advantageous behavior has led to a proposal that central representations of bodily states of arousal guide behavior and bias decision-making, formulated as the "somatic marker hypothesis" (Damasio et al., 1991; Damasio, 1994). These background data provided the focus for the present study, where the specific aim was to examine the influence of uncertainty (risk) and anticipatory autonomic arousal on delay period activity prior to the outcome of a reward-related decision. In our study, it is important to note that activity during the delay period cannot be solely attributed to anticipation of reward-related feedback, and includes non-specific processes such as attention, working memory, motor behavior, and anticipation (independent of the reward component). However, to disambiguate brain activity related to cognitive and autonomic aspects of motivational behavior, we examined for specific modulation of delay period activity by representations of uncertainty and anticipatory arousal (indexed by GSR) prior to the outcome of a reward-related decision.

Activity in bilateral orbitofrontal cortex and anterior cingulate was modulated as a function of outcome uncertainty. Previous studies have implicated orbitofrontal cortex in the processing and flexible representation of reward-related information. In nonhuman primates, neurons within orbitofrontal cortex respond to rewardexpectancy and receipt of reward, and differentiate 
$x=8 \mathrm{~mm}$

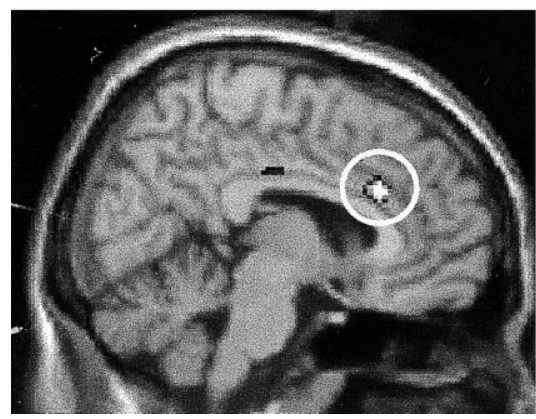

$y=28 \mathrm{~mm}$

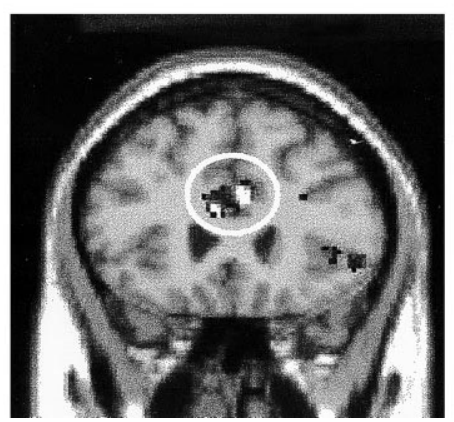

$z=24 \mathrm{~mm}$

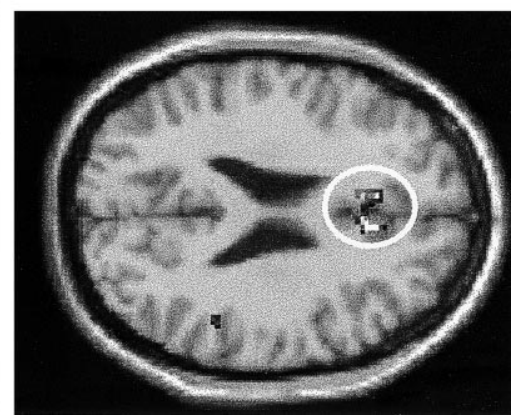

Figure 5. Modulation of Anticipatory Delay Period Activity by Both Risk and Arousal

The figure illustrates group data on parasaggittal, axial, and horizontal sections of a template image showing bilateral anterior cingulate regions where there was a significant modulation of delay period activity by both uncertainty and anticipatory arousal. Horizontal (x), lateral $(y)$, and vertical $(z)$ distances from the anterior commissure are given above the sections. For illustrative purposes, data is displayed at $p<$ 0.001 (uncorrected).

between stimuli associated with reward of differing magnitude (Critchley and Rolls, 1996a, 1996b; Rolls et al., 1996; Tremblay and Schultz, 1999, 2000). Neuroimaging studies in humans also suggest that orbitofrontal cortex, including lateral regions, are involved in processing information about risk and reinforcement (Rogers et al., 1999; Elliott et al., 2000a, 2000b). In the present study, we show that orbitofrontal cortex activity increases with increasing outcome uncertainty. Thus, our findings provide evidence implicating human orbitofrontal cortex in representation of outcome expectancy, where outcome is a proxy for reward. Moreover, maintenance of riskrelated information within orbitofrontal cortex prior to outcome may provide the necessary context for rewardrelated learning prospectively to bias future behavior.

Although we observed modulation of lateral orbitofrontal cortex by outcome uncertainty, human lesion studies also implicate medial orbitofrontal and ventromedial prefrontal cortices in representations of reward and risk (Rolls et al., 1994; Bechara et al., 1996, 1997). Medial orbitofrontal cortex, particularly caudally, is susceptible to fMRI signal dropout; thus we are limited in inferring whether this region contributes significantly to the representation of risk in the present study. In the adjacent anterior cingulate cortex, however, activity was modulated bilaterally as a function of the degree of outcome uncertainty. Previous functional imaging studies have implicated anterior cingulate cortex in visual attention (Nobre et al., 1997), emotional attention (Lane et al., 1997), anticipatory anxiety (Chua et al., 1999), monitoring cognitive conflicts (MacDonald et al., 2000), processing reward-related conflicts (Rogers et al., 1999), and performing difficult cognitive tasks (Paus et al., 1998). Our own observation of increased anterior cingulate activity with increasing outcome uncertainty is consistent with many of these proposed cognitive roles for the anterior cingulate because outcome uncertainty (risk) embodies elements of conflict, anxiety, anticipation, and attention.

Delay period activity in right dorsolateral prefrontal cortex and anterior cingulate was modulated as a function of the degree of anticipatory arousal (measured with GSR). Dorsolateral prefrontal cortex has been implicated in both animal and human experiments in working memory (i.e., the retention of information over short delays for subsequent behavioral use) (e.g., Fuster, 1973; Funahashi et al., 1989; Petrides et al., 1993; GoldmanRakic, 1996; Smith et al., 1996; Courtney et al., 1997; Postle and D'Esposito, 1999). Delay period activity in dorsolateral prefrontal cortex is also modulated as a function of reward magnitude (Watanabe, 1996; Leon and Shadlen, 1999). In our study, activity in dorsolateral prefrontal cortex corresponded with the level of physiological arousal rather than more cognitive attributes of the experimental trials (i.e., degree of risk associated with the prior decision). In our view, it is likely that the magnitude of expected reward will be closely related to the degree of bodily arousal. Recent evidence also suggests that activity in dorsolateral prefrontal cortex during delay in a spatial working memory task may reflect attentional selection (Miller, 1999) rather than maintenance per se (Rowe et al., 2000). Moreover, dorsolateral prefrontal cortex, like the anterior cingulate, is a component of an attentional network (e.g., Nobre et al., 1997). In this context, it is notable that GSR variability has been used widely as an index of attention (Bouscein, 1992). We suggest that our findings are consistent with evidence indicating shared neural substrates for central representation of autonomic arousal and attention (Critchley et al., 2000a).

The anterior cingulate cortex is implicated in many emotional and cognitive processes (Vogt et al., 1992). In the present study, during the delay period, the magnitude of anterior cingulate activity strongly reflected the degree of anticipatory arousal (indexed by GSR). Previous studies have shown both impairment of GSR following anterior cingulate lesions (Zahn, Grafman, and Tranel, 1999; Tranel, 2000) and a correlation between GSR and anterior cingulate activity (Fredrikson et al., 1998). Galvanic skin conductance response can also be modulated by direct electrical stimulation of anterior cingulate (Mangina and Beuzeron-Mangina 1996). Anterior cingulate activity has also been correlated with cardiovascular measures of sympathetic arousal (Critchley et al., $2000 \mathrm{~b}$ ), and we have also reported, in an earlier fMRI study, an association between GSR and activity in medial prefrontal regions rostral to, but intimately 
connected to, the identified anterior cingulate area (Critchley et al., 2000a). In the latter study, GSR-related anterior cingulate activity was not seen because, in part, such regions as anterior cingulate were masked from the analysis because they showed task-related activity and could not uniquely be attributed to autonomic responses. In the present study, the observation that anterior cingulate activity reflects anticipatory arousal (indexed by GSR) suggests that this region is involved in anticipatory somatic responses as well as the representation of outcome uncertainty described above. Thus activity within this region is implicated in mediating cognitive and somatic dimensions of anticipation, as exemplified by the observation (using a conjunction analysis) of enhanced activity with increasing magnitude of anticipatory GSR. Thus, the same subregion of anterior cingulate represents both cognitive uncertainty and physiological arousal, a finding that adds further weight to the proposal that a function of anterior cingulate is to integrate motivationally important information with appropriate bodily responses (Critchley et al., 2000b).

In summary, we report activity during the delay period before the outcome of reward-related decisions in brain areas implicated in the control of social and emotional behavior. Moreover, activity in anterior cingulate during this period was modulated by both outcome risk and anticipatory arousal, with discrete regions of cingulate reflecting both. Our findings strongly support a role for anterior cingulate in integrating cognitive processing of uncertainty with adaptive changes in bodily state that may serve to prospectively bias behavior.

\section{Experimental Procedures}

\section{Subjects}

Eight healthy right-handed subjects (six male, two female, mean age 29.2 years [SD 4.1]) volunteered for participation in a study approved by local ethics committee, and gave full informed consent. Prior to scanning, subjects were screened to exclude medical conditions or medication effects that might affect brain function.

\section{Experimental Task}

Each subject performed a decision-making task with feedback for which they obtained monetary gain or loss. Stimuli were playing cards numbered from 1 to 10 (excluding face cards) visually presented on a screen subtending $20^{\circ}$ of the subject's visual field. Each trial began with a $0.5 \mathrm{~s}$ presentation of a cue (the word "new") followed by a $1 \mathrm{~s}$ presentation of a playing card (the cue card). Subjects were required to indicate by an immediate button press response if they thought the next card would be higher or lower than the cue card. There then followed a delay period ( $8.5 \mathrm{~s}$ ) before a second playing card (feedback card) was presented for $1 \mathrm{~s}$; this card provided feedback about the earlier decision. Correct decisions were associated with monetary gain, and incorrect decisions with monetary loss for the subject (Figure 1). The next trial began $8 \mathrm{~s}$ after presentation of the feedback card. Subjects were presented with a total of 100 trials. We used a long delay period to limit the extent to which GSR responses to the cue stimulus/decision would confound the measured anticipatory arousal prior to feedback. Similarly, a long intertrial interval was used to allow GSR to approach baseline before the start of each trial. Each subject received the same randomized stimulus presentation, with ten presentations of each cue card over the course of the experiment. Feedback cards were pseudorandomized with the constraints that in any trial the feedback card was always higher or lower than the cue card, and that the probability of the cue card being higher or lower approximated to the true likelihood for a random set. If the face value of the cue card was 1 or 10 , the subject would know the correct response with certainty, while the remaining values of cue cards carried different degrees of risk (e.g., there was a $56 \%$ probability that a cue card face value 5 (high risk) would be followed by a higher card and an $89 \%$ probability that a cue card face value 2 (low risk) would be followed by a higher feedback card) (Table 1). It was never possible for subjects to predict the values of feedback cards beyond the probability of the next card being higher or lower. The financial consequence of each trial was only conveyed to the subject by the face value of the feedback card, which indicated whether the prior decision had been successful or not and, hence, if the subject had won or lost money on that trial. The subject was not kept informed about overall wins and losses during the task. Subjects were told that they would receive a financial reward (50 p) for correct answers and lose twice this amount (£1) for wrong answers, from a starting point of zero. This particular pattern of payoff was chosen to allow subjects to win approximately $£ 20$ over the course of the experiment and, by punishing wrong responses more than rewarding right responses, to maintain interest in achieving correct answers and to minimize illogical response strategies.

\section{Data Acquisition}

Subjects were scanned during task performance using a Siemens VISION system (Ehrlangen, Germany) at 2 Tesla to acquire gradientecho, echoplanar T2*-weighted images with BOLD (blood oxygenation level dependent) contrast. Each volume comprised $32 \times 3$ $\mathrm{mm}$ axial scans with 3-mm in-plane resolution and volumes were continuously acquired every $3.17 \mathrm{~s}$. Subjects were placed in light head restraint within the scanner to limit head movement during data acquisition. Each run began with 6 "dummy" volumes (subsequently discarded) to allow for T1 equilibration effects. Additionally a T1weighted structural image was acquired in each subject for registration with the functional BOLD data. This scan was also used to determine the extent of regional BOLD signal dropout from each subject's functional data set. In all subjects, echoplanar T2*weighted images provided excellent coverage throughout the brain. However, some anticipated signal dropout was observed in frontal pole and in posterior orbitomedial prefrontal cortex (regions of air and tissue boundaries), limiting our inferences about task-related activity in these regions.

Galvanic skin conductance responses were continuously monitored (SCL 200, Biofeedback Systems, Manchester, UK) from the palmar surface of index and third fingers of left hand, and the amplified analog output of the GSR sampled at $100 \mathrm{~Hz}$ and stored using Spike2 software (Cambridge Electronic Design, Ltd., Cambridge, UK). Offline filtering and smoothing was used to remove scanning related noise from GSR traces. For each subject, the skin conductance trace was normalized to mean (Critchley et al., 2000a).

\section{Image Coregistration, Realignment, and Normalization}

Data preprocessing to was performed using Statistical Parametric Mapping (SPM99; Wellcome Department of Cognitive Neurology, London, UK; http://www.fil.ion.ucl.ac.uk/spm) to correct for head movement and allow functional data sets to be entered into group analyses. All functional volumes, independent of session or paradigm, were realigned to the first volume acquired using rigid-body registration (Friston et al., 1995a) and a mean realigned volume created. Sessions containing obvious movement artifacts were discarded at this stage. The subject's T1-weighted structural scan was coregistered to the mean functional volume; the mean volume was used to determine the parameters applied to all volumes during spatial normalization and resampling (Ashburner et al., 1997; Ashburner and Friston, 1999) to a standard template. As the volume of brain sampled in each study was affected by the position of the subject within the scanner's field of view, we found that the extreme superior and inferior portions of the subject's brain were sparsely sampled. To address this, voxels not sampled in every session were eliminated during normalization. All functional volumes were then smoothed with an 8-mm FWHM Gaussian kernel. Global changes in $\mathrm{FMRI}$ response from scan to scan were removed by proportionally scaling each scan to have a common global mean voxel value.

\section{Statistical Analysis}

Data were analyzed using SPM99 employing the general linear model, where statistical inferences were based on the theory of 
random Gaussian fields (Friston et al., 1995a, 1995b, 1995c). A design matrix was constructed for subject-by-condition analysis of event and epoch related activity. Presentations of the cue card and feedback card were treated as events and entered into the design as separate columns. The $8.5 \mathrm{~s}$ delay between the cue and feedback cards was treated as an epoch. A canonical hemodynamic response function was used as the basis set for individual events and convolved with a boxcar for analysis of epochs. This effectively implemented a least-squares deconvolution of enduring components of the hemodynamic response to individual events and epochs to allow examination of regional changes in neural activity underlying these hemodynamic responses. Neural activity during the anticipatory delay period was examined for (1) main effect of epoch, (2) parametric modulation by risk (uncertainty at the time of decision), (3) parametric modulation by sympathetic arousal (indexed by mean GSR during $4 \mathrm{~s}$ period before feedback), and (4) the interaction between risk and arousal; (5) we also tested for delay period activity commonly modulated by both risk and arousal, using conjunction analyses (Price and Friston, 1996; Friston et al., 1999). Mean-corrected regressors for parametric modulation of epoch-related activity were entered into the design matrix. Anticipatory arousal relative to each trial was derived from the mean GSR in the last $4 \mathrm{~s}$ of the epoch, thereby accommodating delay between central neural activity and peripheral GSR response, and providing consistency with other studies of anticipatory arousal (Bechara et al., 1996, 1997). To avoid the potential confounds arising from correlations between our measure of arousal and the risk, we orthogonalized arousal (indexed by GSR) with respect to risk, thereby excluding common variance and allow examination of interactions between orthogonalized variables. Statistical parametric maps (SPMs) were based on a multiple regression analysis. Subject-specific low-frequency confounds were removed in the regression. High-frequency noise was also removed using a Gaussian low-pass filter (full width half-maximum, $4 \mathrm{~s}$ ), and global differences were controlled by proportional scaling. The analysis of parametric modulation of delay-related activity prior to feedback was performed using regression analyses equivalent to multiplying ("modulating") the main effect of the delay period with the regressors for measures of risk, arousal, and their interaction. The $p$ values associated with these modulatory effects are mathematically identical to the corresponding partial correlation coefficients. The significance of the association between the observed time series and one or a linear combination of these regressors is tested with the $t$ statistic to give an SPM $\{t\}$. Significant regional brain activity relating to task, risk, and arousal was determined using conjunction analyses across subjects (Friston et al., 1999) within a fixed effects model. This allows inference that every subject activated these brain regions and that these activations would be present in a substantial proportion of the population (Friston et al., 1999). We report voxel clusters of ten or more, reaching statistical significance at $p<0.05$ (corrected for whole brain and, for predicted regions of interest, thresholded at $p<0.01$ [uncorrected] then reported if significance survives $p<0.05$ [corrected] for small volume of region of interest) (Worsley et al., 1996). Descriptions of anatomical location were determined using average structural MRIs normalized to standard space (Talairach and Tournoux, 1988), using the atlas of Duvernoy (1991).

\section{Acknowledgments}

This work was supported by the Wellcome Trust. R. J. D. is supported by a program grant from the Wellcome Trust. H. D. C. is supported by a Brain Research Trust fellowship. We are very grateful to Karl Friston for helpful advice.

Received June 12, 2000; revised November 21, 2000.

\section{References}

Adolphs, R., Tranel, D., and Damasio, A.R. (1998). The human amygdala in social judgment. Nature 393, 470-474.

Ashburner, J., and Friston, K. (1999). Nonlinear spatial normalization using basis functions. Hum. Brain Map. 7, 254-266.

Ashburner, J., Neelin, P., Collins, D.L., Evans, A.C., and Friston,
K. (1997). Incorporating prior knowledge into image registration. Neurolmage 6, 344-352.

Bechara, A., Tranel, D., Damasio, H., and Damasio, A.R. (1996). Failure to respond autonomically to anticipated future outcomes following damage to prefrontal cortex. Cereb. Cortex 6, 215-225.

Bechara, A., Damasio, H., Tranel, D., and Damasio, A.R. (1997). Deciding advantageously before knowing the advantageous strategy. Science 275, 1293-1295.

Bechara, A., Damasio, H., Damasio, A.R., and Lee, G.P. (1999). Differential contributions of the human amygdala and ventromedial prefrontal cortex to decision-making. J. Neurosci. 19, 5473-5481.

Bouscein, W. (1992). Electrodermal Activity (New York: Plenum). Chua, P., Krams, M., Toni, I., Passingham, R., and Dolan, R. (1999). A functional anatomy of anticipatory anxiety. Neurolmage 9, 563-571. Courtney, S.M., Ungeleider, L.G., and Haxby, J.V. (1997). Transient and sustained activity in a distributed neuronal system for human working memory. Nature 386, 608-611.

Critchley, H.D., and Rolls, E.T. (1996a). Olfactory neuronal responses in the primate orbitofrontal cortex: Analysis in an olfactory discrimination task. J. Neurophysiol. 75, 1659-1672.

Critchley, H.D., and Rolls, E.T. (1996b). Hunger and satiety modify the responses of olfactory and visual neurons in the primate orbitofrontal cortex. J. Neurophysiol. 75, 1673-1686.

Critchley, H.D., Elliott, R., Mathias, C.J., and Dolan, R.J. (2000a). Neural activity relating to the generation and representation of galvanic skin conductance response: a functional magnetic imaging study. J. Neurosci. 20, 3033-3040.

Critchley, H.D., Corfield, D.R., Chandler, M.P., Mathias, C.J., and Dolan, R.J. (2000b). Cerebral correlates of autonomic cardiovascular arousal: a functional neuroimaging investigation. J. Physiol. 523, 259-270.

Damasio, A.R., Tranel, D., and Damasio, H.C. (1990). Individual with sociopathic behavior caused by frontal damage fail to respond autonomically to social stimuli. Behav. Brain Res. 41, 81-94.

Damasio, A.R., Tranel, D., and Damasio, H.C. (1991). Somatic markers and the guidance of behavior: theory and preliminary testing. In: Frontal Lobe Function and Dysfunction, H.S. Levin, H.B. Eisenberg, and L.B. Benton, eds. (New York: Oxford University Press), pp. 217-229.

Damasio, A.R. (1994). Descartes' Error. (New York: Putnam).

Duvernoy, H.M. (1991). The Human Brain: Surface Three Dimensional Sectional Anatomy and MRI (New York: Springer-Verlag).

Elliott, R., Dolan, R.J., and Frith, C.D. (2000a). Dissociable functions in medial and lateral orbitofrontal cortex. Evidence from human neuroimaging studies. Cereb. Cortex 10, 308-317.

Elliott, R., Friston, R.J., and Dolan, R.J. (2000b). Dissociable responses associated with reward, punishment and risk-taking behavior. J. Neurosci. 20, 6159-6165.

Eslinger, P.J., and Damasio, A.R. (1985). Severe disturbance of higher cognition after bilateral frontal lobe ablation: patient E.V.R. Neurology 35, 1731-1741.

Fredrikson, M., Furmark, T., Olsson, M.T., Fischer, H., Andersson, J., and Langstrom, B. (1998). Functional neuroanatomical correlates of electrodermal activity: a positron emission tomographic study. Psychophysiology 35, 179-185.

Friston, K., Ashburner, J., Frith, C.D., Poline, J.-B., Heather, J.D., and Frackowiak, R.S.J. (1995a). Spatial registration and normalization of images. Hum. Brain Map. 2, 165-189.

Friston, K., Holmes, A.P., Worsley, K., Poline, J.-B., Frith, C., and Frackowiak, R.S.J. (1995b). Statistical parametric maps in functional imaging: a general linear approach. Hum. Brain Map. 2, 189-210.

Friston, K.J., Holmes, A.P., Poline, J.-B., Grasby, P.J., Williams, S.C., Frackowiak, R.S., and Turner, R. (1995c). Analysis of fMRI timeseries revisited. Neurolmage 1, 45-53.

Friston, K.J., Holmes, A.P., Price, C.J., Buchel, C., and Worsley, K.J. (1999). Multisubject fMRI studies and conjunction analyses. Neurolmage 10, 385-396.

Funahashi, S., Bruce, C.J., and Goldman-Rakic, P.S. (1989). Mne- 
monic coding of visual space in the monkey's dorsolateral prefrontal cortex. J. Physiol. 61, 331-338.

Fuster, J.M. (1973). Unit activity in prefrontal cortex during delayedresponse performance: neuronal correlates of transient memory. J. Physiol. 36, 61-78.

Goldman-Rakic, P. (1996). Regional and cellular fractionation of working memory. Proc. Natl. Acad. Sci. USA 93, 13435-13437.

Lane, R.D., Fink, G.R., Chau, P.M., and Dolan, R.J. (1997). Neural activation during selective attention to subjective emotional responses. NeuroReport 8, 3969-3972.

Leon, M.I., and Shadlen, M.N. (1999). Effect of expected reward magnitude on the response of neurons in the dorsolateral prefrontal cortex of the macaque. Neuron 24, 415-425.

MacDonald, A.W., Cohen, J.D., Stenger, V.A., and Carter, C.S. (2000). Dissociating the role of the dorsolateral prefrontal and anterior cingulate cortex in cognitive control. Science 288, 1835-1838.

Mangina, C.A., and Beuzeron-Mangina, J.H. (1996). Direct electrical stimulation of specific human brain structures and bilateral electrodermal activity. Int. J. Psychophysiol. 22, 1-8.

Miller, E.K. (1999). The prefrontal cortex: complex neural properties for complex behavior. Neuron 22, 15-17.

Nobre, A.C., Sebestyen, G.N., Gitelman, D.R., Mesulam, M.M., Frackowiak, R.S.J., and Frith, C.D. (1997). Functional localization of the system for visuospatial attention using positron emission tomography. Brain 120, 515-533.

Paus, T., Koski, L., Caramanos, Z., and Westbury, C. (1998). Regional differences in the effects of task difficulty and motor output on blood flow response in the human anterior cingulate cortex: a review of 107 PET activation studies. NeuroReport 9, R37-R47.

Petrides, M., Alivisatos, E., Meyer, E., and Evans, A.C. (1993). Functional activation of the human frontal cortex during performance of verbal working memory tasks. Proc. Natl. Acad. Sci. USA 90, 878-882.

Postle, B.R., and D'Esposito, M. (1999). "What"-then-“Where" in visual working memory: an event-related fMRI study. J. Cog. Neurosci. $11,585-597$

Price, C.J., and Friston, K. (1996). Cognitive conjunctions: a new approach to brain activation experiments. Neuroimage 5, 261-270.

Rogers, R.D., Owen, A.O., Middleton, H.C., Williams, E.J., Pickard, J.D., Sahakian, B.J., and Robbins, T.W. (1999). Choosing between small, likely rewards and large, unlikely rewards activates inferior and orbital prefrontal cortex. J. Neurosci. 20, 9029-9038.

Rolls, E.T., Hornak, J., Wade, D., and McGrath, J. (1994). Emotionrelated learning in patients with social and emotional changes associated with frontal lobe damage. J. Neurol. Neurosurg. Psychiatry 57, 1518-1524.

Rolls, E.T., Critchley, H.D., Mason, R., and Wakeman, E.A. (1996). Orbitofrontal cortex neurons: role in olfactory and visual association learning. J. Neurophysiol. 75, 1970-1981.

Rowe, J.B., Toni, I., Josephs, O., Frackowiak, R.S.J., and Passingham, R.E. (2000). The prefrontal cortex: response selection or maintenance within working memory? Science 288, 1656-1664.

Saver, J.L., and Damasio, A.R. (1991). Preserved access and processing of social knowledge in a patient with acquired sociopathy due to ventromedial frontal damage. Neuropsychologia 29, 12411249 .

Shallice, T., and Burgess, P.W. (1991). Deficits in strategy application following frontal lobe damage in man. Brain 114, 727-741.

Smith, E.E., Jonides, J., and Koeppe, R.A. (1996). Dissociating verbal and spatial working memory using PET. Cereb. Cortex 6, 11-20.

Talairach, J., and Tournoux, P. (1988). Co-planar Stereotaxic Atlas of the Human Brain. (Stuttgart, Germany: Theime).

Tranel, D., and Damasio, H. (1994). Neuroanatomical correlates of electrodermal skin conductance responses. Psychophysiology 31 , 427-438.

Tranel, D. (2000). Electrodermal activity in cognitive neuroscience: neuroanatomical and neuropsychological correlates. In: Cognitive
Neuroscience of Emotion, R.D. Lane and L. Nadel, eds. (New York Oxford University Press), pp. 192-224.

Tremblay, L., and Schultz, W. (1999). Relative reward preference in primate orbitofrontal cortex. Nature 398, 704-708.

Tremblay, L., and Schultz, W. (2000). Reward-related neuronal activity during go-nogo task performance in primate orbitofrontal cortex J. Neurophysiol. 83, 1864-1876.

Vogt, B.A., Finch, D.M., and Olson, C.R. (1992). Functional heterogeneity in cingulate cortex: the anterior executive and posterior evaluative regions. Cereb. Cortex 6, 435-443.

Watanabe, M. (1996). Reward expectancy in primate prefrontal neurons. Nature 382, 629-632.

Worsley, K.J., Marrett, S., Neelin, P., Vandal, A.C., Friston, K., and Evans, A.C. (1996). A unified statistical approach for determining significant signals in images of cerebral activation. Hum. Brain Map. 4, 58-73.

Zahn, T.P., Grafman, J., and Tranel, D. (1999). Frontal lesions and electrodermal activity: effects of significance. Neuropsychologica $37,1227-1241$ 